\title{
Paraparesia transitoria como manifestación de una estenosis carotídea izquierda
}

\author{
J. Bruna-Escuer, A. Escrig-Avellaneda, L. Romero-Pinel, M. Jato-de Evan, \\ S. Martínez-Yélamos, F. Rubio-Borrego
}

\section{TRANSIENT PARAPARESIS AS A MANIFESTATION OF LEFT CAROTID STENOSIS}

\begin{abstract}
Summary. Introduction. Paraparesis caused by a cerebrovascular disorder is infrequent, although it is seen in infraction of both anterior cerebral arteries (ACA), in vertebrobasilar insufficiency syndromes or in infarction of the border zones of the anterior circulation. Case report. Male aged 52 years, right-handed, with a history of high blood pressure, who visited because of two episodes of transient paraparesis that lasted 5 minutes and 15 hours. During the last episode the presence of paraparesis and left Babinski sign were observed. Complementary explorations conducted to test for medullar pathology were negative. Cranial MRI showed only bilateral lacunar infarctions in deep territories. Four months later, the patient presented an episode of motor aphasia and paresthesia of the right lower limb, which remitted spontaneously in 10 minutes. Echo-Doppler exploration of the supra-aortic trunks showed significant stenosis of the left internal carotid artery (LICA) and occlusion of the right artery (RICA). Arteriography of the supra-aortic trunks revealed a 99\% stenosis of the RICA and 95\% of the LICA, with vascularisation of both ACA dependent on the LICA. A left carotid endarterectomy was performed and the patient has remained asymptomatic to date. Conclusion. In our patient, both ACA depended on the flow from the LICA. We therefore consider that the symptoms of transient paraparesis were secondary to the left carotid stenosis, either due to a haemodynamic or an artery to artery embolic mechanism. [REV NEUROL 2003; 36: 933-5]
\end{abstract}

Key words. Anterior cerebral artery. Carotid stenosis. Paraparesis.

\section{INTRODUCCIÓN}

La circulación cerebral puede presentar tanto variantes anatómicas como alteraciones funcionales del flujo sanguíneo secundarias a situaciones patológicas. Por ello, en ocasiones, la enfermedad cerebrovascular puede manifestarse con una clínica atípica que induzca a falsas localizaciones lesionales. La paraparesia de presentación brusca es una manifestación poco frecuente de la patología vascular cerebral. Suele observarse en la isquemia del territorio de ambas arterias cerebrales anteriores (ACA) [1], normalmente en el contexto de un vasoespasmo por rotura de aneurismas de la arteria comunicante anterior ACA [2]. También puede aparecer un cuadro de paraparesia en la isquemia vertebrobasilar, secundaria a fenómenos de robo desde la circulación posterior hacia la circulación anterior [3-5]. Incluso se ha descrito en la isquemia bihemisférica de territorios frontera entre las ACA y las medias $[1,6]$. En los dos últimos casos, suele tratarse de pacientes portadores de estenosis carotídeas bilaterales graves, lo que constituye el $0,9-2,4 \%$ [6] de los pacientes con patología cerebral vascular. Presentamos a continuación un caso de paraparesia transitoria como primera manifestación de una estenosis carotídea izquierda (ECI).

\section{CASO CLÍNICO}

Varón de 52 años, diestro, con antecedentes de hipertensión arterial de 10 años de evolución y hábito de consumo alcohólico moderado, que consultó por dos episodios transitorios de paraparesia, de 5 minutos y de 15 horas de evolución.

Recibido:23.10.02. Recibido en versión revisada:24.02.03. Aceptado:03.03.03. Servicio de Neurología. Ciutat Sanitària i Universitària de Bellvitge. Universitat de Barcelona. L'Hospitalet del Llobregat, Barcleona, España.

Correspondencia: Dr. Jordi Bruna Escuer. Servicio de Neurología. Ciutat Sanitària i Universitària de Bellvitge. Universitat de Barcelona. Feixa Llarga, s/n. E-08907 L'Hospitalet del Llobregat, Barcelona. Fax: +34 932607882. E-mail:35078jbe@comb.es

(C) 2003, REVISTA DE NEUROLOGÍA
En su exploración destacaba una tensión arterial de 230/110, paraparesia 4/ 5 bilateral, Babinski izquierdo, apraxia constructiva y conducta desinhibida. Las exploraciones complementarias practicadas para el estudio de patología medular fueron normales. El ECG y la radiografía de tórax fueron normales. La analítica general, VSG, proteinograma, serología en sangre de VIH, sífilis, CMV, herpes simple y Epstein Barr, riesgo trombótico, ANA, antiADN, anticardiolipinas, anticuerpos anti- $\beta$-2-glicoproteína I y ANCA, fueron negativos. En el líquido cefalorraquídeo, la determinación de células, proteínas, glucosa, bandas oligoclonales y serologías, también fueron normales. Una RM craneal mostró únicamente imágenes focales de pequeño tamaño, compatibles con lesiones isquémicas en la sustancia blanca supratentorial y los ganglios basales. No se realizó arteriografía medular.

Cuatro meses después, el paciente presentó, sin relación con el ortostatismo ni el ejercicio, un episodio de afasia motora y parestesias en la extremidad inferior derecha, que se autolimitó en 10 minutos. La ecografía Doppler de los troncos supraórticos mostró una estenosis significativa de la carótida interna izquierda (CII) y una oclusión de la carótida interna derecha (CID). La arteriografía de los troncos supraórticos objetivó una estenosis de1 99\% de la CID (Fig. 1) y del 95\% de la CII (Fig. 2). Ambas ACA se rellenaban a través de la CII (Fig. 3). La arteria vertebral derecha era hipoplásica y la vertebral izquierda vascularizaba el territorio posterior sin anomalías. Se practicó una endarterectomía carotídea izquierda, y el paciente permaneció asintomático hasta la fecha.

\section{DISCUSIÓN}

La paraparesia intermitente en patología vascular, habitualmente plantea el diagnóstico diferencial entre fístula arteriovenosa o accidente isquémico transitorio (AIT) de arterias medulares [5]. Los pacientes con estenosis graves de ambas carótidas internas, cuando se enfrentan a una situación de bajo gasto, pueden presentar paraparesia como manifestación de isquemia de territorios frontera de la circulación anterior [6]. En un 10\% de la población se encuentra una aplasia/hipoplasia grave del segmento A1 de la ACA, de tal forma que ambas ACA se nutren de la misma carótida. En estos casos, una estenosis grave en dicha carótida, o un embolismo sobre la única ACA funcionante produciría síntomas en el territorio de ambas ACA. El embo- 


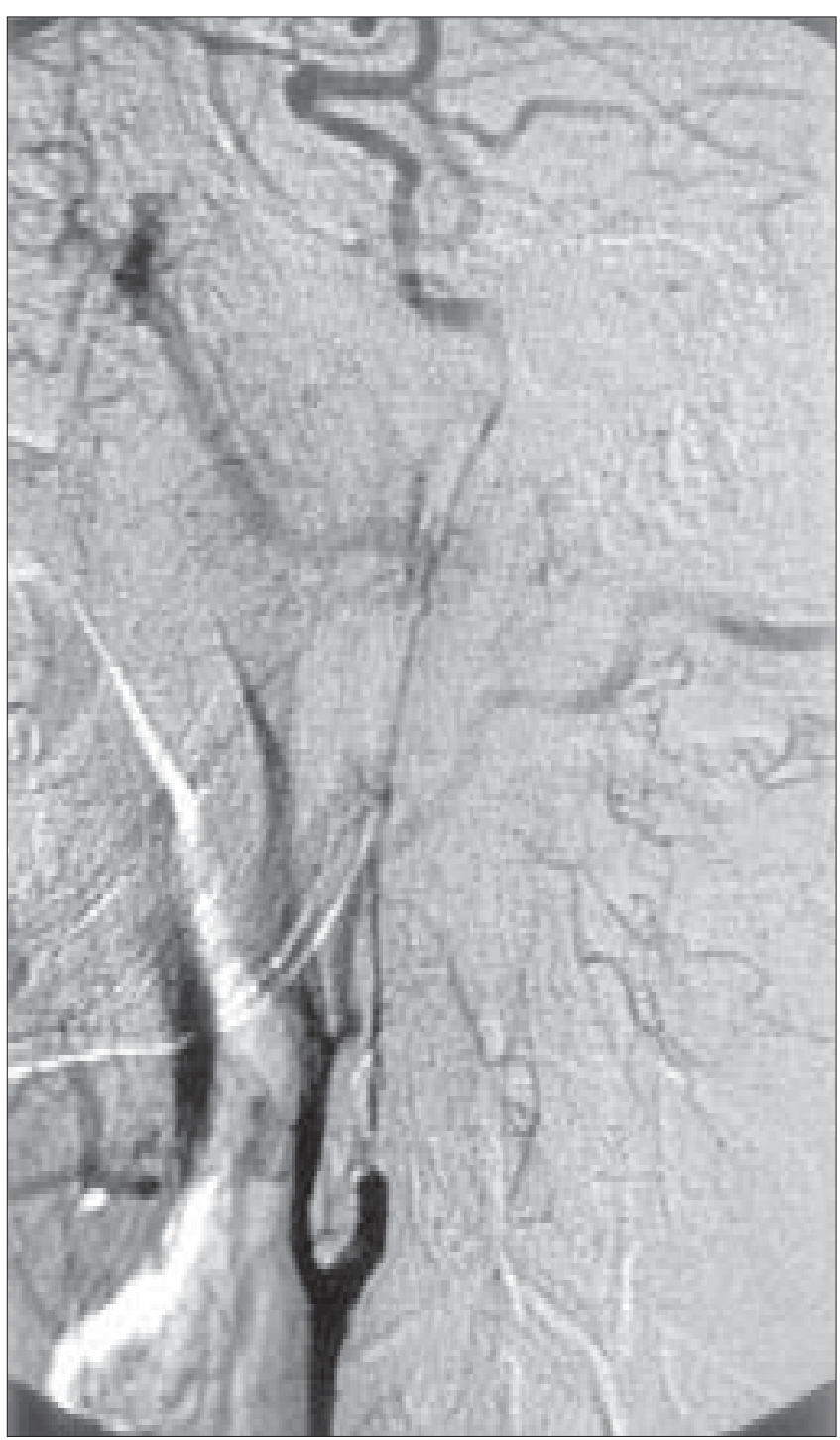

Figura 1. Arteria carótida interna derecha con estenosis del 99\%, que distribuye el flujo hacia el territorio silviano derecho y el cerebral posterior a través de la comunicante posterior.

lismo procedente de las arterias carótidas, junto con el embolismo cardíaco, es la causa más frecuente de infarto de las ACA [1]. Otros autores $[1,4,5]$ describen en este tipo de pacientes un robo preferencial hacia la circulación anterior desde la circulación posterior. En nuestro caso, la arteriografía no muestra un flujo invertido hacia territorios anteriores desde la circulación vertebrobasilar, y ambas ACA dependen de la CII. Probablemente, en nuestro paciente la paraparesia transitoria fue secundaria a la ECI, bien por un mecanismo hemodinámico o embólico arteria-arteria.

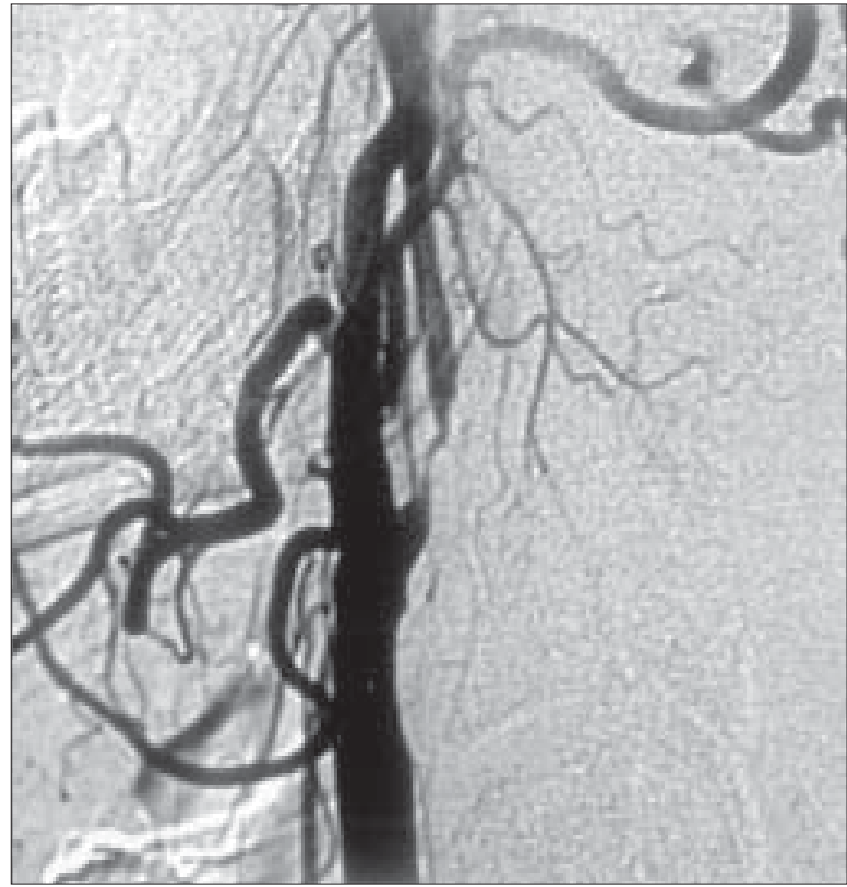

Figura 2. Arteria carótida izquierda con estenosis del $95 \%$.

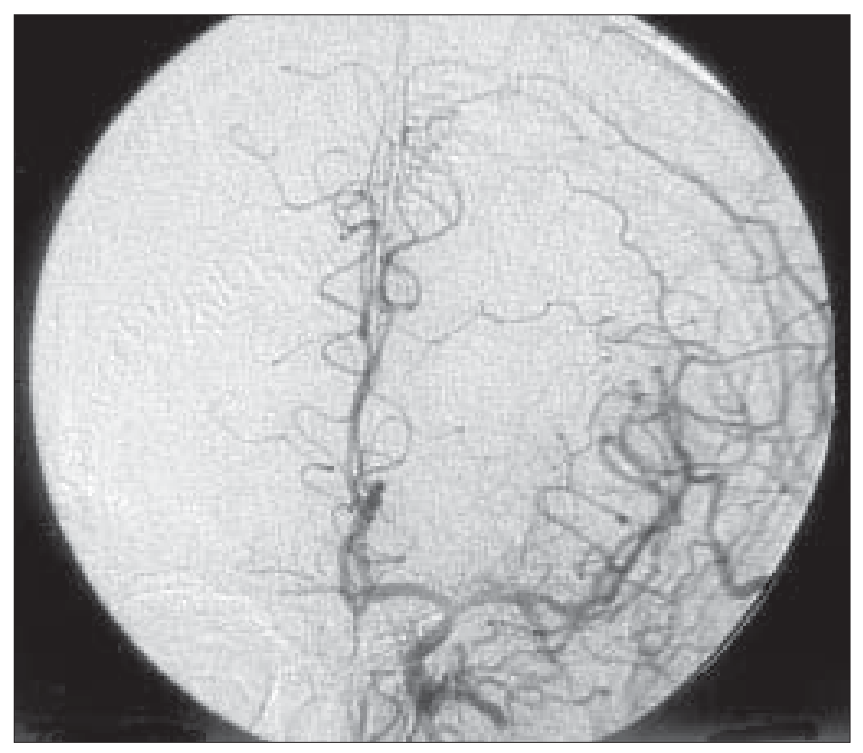

Figura 3. Arterias cerebrales anteriores, con flujo de ambas dependiente de la arteria carótida interna izquierda.

Así, se recomienda la realización de una ecografía Doppler de los troncos supraórticos en el estudio de pacientes con paraparesia transitoria de etiología no establecida, sobre todo si las exploraciones cognitivas muestran alteraciones de las funciones corticales.

\section{BIBLIOGRAFÍA}

1. Brust J, Sawada T, Kazui S. Anterior cerebral artery. In Bogousslavsky J, Caplan L, eds. Stroke syndromes. 2 ed. New York: Cambridge University Press; 2001. p. 439-50.

2. Gacs G, Fox AJ, Barnett HJM, Vinuela F. Occurrence and mechanism of occlusion of the anterior cerebral artery. Stroke 1983; 14: 952-9.

3. Bogousslavsky J, Regli F. Vertebrobasilar transient ischemic attacks in internal carotid artery occlusion or tight stenosis. Arch Neurol 1985; 42: 64-8.
4. Swanson PD, Calanchini PR, Dyken ML. A Cooperative study of hospital frequency and character of transient ischemic attacks: II. Performance of angiographic among six centers. JAMA 1977; 237: 2202-6.

5. Ueda K, Toole JF, McHenry LC. Carotid and vertebrobasilar transient ischemic attacks: Clinical and angiographic correlation. Neurology 1979; 29: 1094-101.

6. Sloan M, Haley E. The syndrome of bilateral hemispheric border zone ischemia. Stroke 1990; 21: 1668-73. 


\section{PARAPARESIA TRANSITORIA COMO MANIFESTACIÓN}

DE UNA ESTENOSIS CAROTÍDEA IZQUIERDA

Resumen. Introducción. La paraparesia por afectación vascular cerebral es infrecuente, aunque se observa en infartos de ambas arterias cerebrales anteriores (ACA), en síndromes de insuficiencia vertebrobasilar o en infartos de territorios frontera de la circulación anterior. Caso clínico. Varón de 52 años, diestro, con antecedentes de hipertensión arterial, que consultó por dos episodios transitorios de paraparesia, de 5 minutos y 15 horas de duración. Durante el último episodio, se objetivó lapresencia de una paraparesia y un Babinski izquierdo. Las exploraciones complementarias practicadas para el estudio de patología medular fueronnegativas. UnaRMcranealmostróúnicamenteinfartos lacunares bilaterales en territorios profundos. Cuatro meses después, el paciente presentó un episodio de afasia motora y parestesias de la extremidad inferior derecha, autolimitado en 10 minutos. La ecografía Doppler de los troncos supraórticos reveló una estenosis significativa de carótida interna izquierda (CII) y una oclusión de la derecha (CID). La arteriografía de los troncos supraórticos demostró una estenosis del 99\% de la CID y del $95 \%$ de la CII, con vascularización de ambas ACA dependientes de la CII. Se practicó una endarterectomía carotídea izquierda, y el paciente permaneció asintomático hasta la actualidad. Conclusión. En nuestro paciente, ambas ACA dependían del flujo de la CII. Por ello, consideramos que el cuadro de paraparesia transitoria fue secundario a la estenosis carotídea izquierda, bien por un mecanismo hemodinámico o embólico arteria-arteria. [REV NEUROL 2003; 36: 933-5]

Palabras clave. Arteria cerebral anterior. Estenosis carotídea. Paraparesia.

\section{PARAPARESIA TRANSITÓRIA COMO MANIFESTAÇÃO DA ESTENOSE CAROTÍDEA ESQUERDA}

Resumo. Introdução. A paraparesia por envolvimento vascular cerebral é rara, embora se observe nos enfartes de ambas as artérias cerebrais anteriores (ACA), nas síndromas de insuficiência vertebrobasilar ou nos enfartes dos territórios de fronteira da circulação anterior. Caso clínico. Homem de 52 anos, destro, com antecedentes de hipertensão arterial, que recorreu à consulta por dois episódios transitórios de paraparesia, de 5 minutos e 15 horas de duração respectivamente. Durante o último episódio, objectivou-se a presença de uma paraparesia e de um Babinsky esquerdo. Os exames complementares realizados para o estudo de patologia medular foram negativos. Uma RM craniana mostrou unicamente enfartes lacunares bilaterais em territórios profundos. Após quatro meses, o doente apresentou um episódio de afasia motora e parestesias da extremidade inferior direita, autolimitado, resolvendo em 10 minutos. O eco-Doppler dos troncos supra-aórticos objectivou uma estenose significativa da carótida interna esquerda (CIE) e uma oclusão da direita (CID). A arteriografia dos troncos supra-aórticos revelou uma estenose de $99 \%$ da CID e de 95\% da CIE. Realizou-se uma endarterectomia carotídea esquerda e o doente permaneceu assintomático até à data. Conclusão. No nosso doente, ambas as ACA dependiam do fluxo da CIE, pelo que consideramos que o quadro de paraparesia transitória foi secundário à estenose carotídea esquerda, devido a um mecanismo hemodinâmico ou embólico artéria-artéria. [REV NEUROL 2003; 36: 933-5]

Palavras chave. Artéria cerebral anterior. Estenose carotídea. Paraparesia. 